\title{
Gerencia logística y global
}

Pablo César Ocampo Vélez*

\section{RESUMEN}

La logística es una filosofía que se encarga de aplicar las buenas prácticas en los macroprocesos, procesos, procedimientos, tareas y productos dentro de una organización, con el fin de satisfacer las necesidades del cliente, quien es la razón de ser de la empresa. Existen entes internacionales que velan porque las compañias en todo el mundo utilicen y difundan las diferentes disciplinas logísticas en toda la red de valor y tengan un mismo enfoque estratégico.El objetivo de este artículo es explicar la existencia de procesos y macroprocesos de clase mundial en la logística, gracias a los cuales hay una gran oportunidad de mejora para los empresarios colombianos, con el fin de reestructurar los procesos estratégicos, tácticos y operacionales de cada Unidad de Negocio, para que sean organizaciones más competitivas, que puedan enfrentar los tratados de libre comercio, brindando mayor sostenibilidad y óptimo nivel de servicio.

\section{Palabras Clave}

Cadena de valor

Cadena de abastecimiento

Logística integral

Global Supply Chain Management

Redes de valor

Valor Agregado

Procesos

Macroprocesos

\section{Introducción}

D urante el seguimiento del artículo se argumenta el concepto de logística integral, en que consiste el gerenciamiento de la cadena de abastecimiento global "Global Supply Chain Management" G.S.C.M, con su alcance, estrategias, macroprocesos, procesos de clase mundial liderados por los entes que velan por la difusión de las buenas prácticas a nivel mundial, principios, ventajas y megatendencias que hace que sea competitivo la cadena de valor en las organizaciones.

Ingeniero Industrial Universidad Autónoma de Colombia. Master in Management of Logistics Systems, École Politechnique Federal Du Lausanne, Switzerland. Docente de Planta Facultad de Posgrados Universidad EAN. 


\section{Concepto de logística}

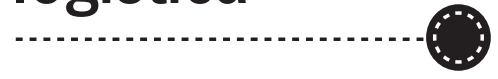

$E_{1}$

Council Supply Chain Management Professionals(CSCMP)esunodelosentes internacionales que describe cómo los procesos de planeación, implementación, control eficiente y efectivo del flujo de materiales e información, recurso humano, almacenaje, servicios, son imperativos para el óptimo desempeño de la cadena logística desde el punto de origen hasta el punto de consumo, satisfaciendo los requerimientos del cliente.

Uno de los paradigmas que cada vez coge más fuerza en el ámbito logístico, es acerca de la creación de valor a través de los eslabones de la cadena de suministro global, donde no solamente con el fin de transformar físicamente una materia prima se genera valor, sino que se debe contemplar diferentes criterios analíticos basados en la necesidad del consumidor final, tales como la cantidad correcta en el lugar indicado y a un adecuado precio basado en la filosofía de justo a tiempo y de la personalización de bienes /servicios.

No sobra señalar que la logística wwno es sólo para la industria, sino para cualquier tipo de organización tal como empresas del gobierno,hospitales,bancos, almacenes

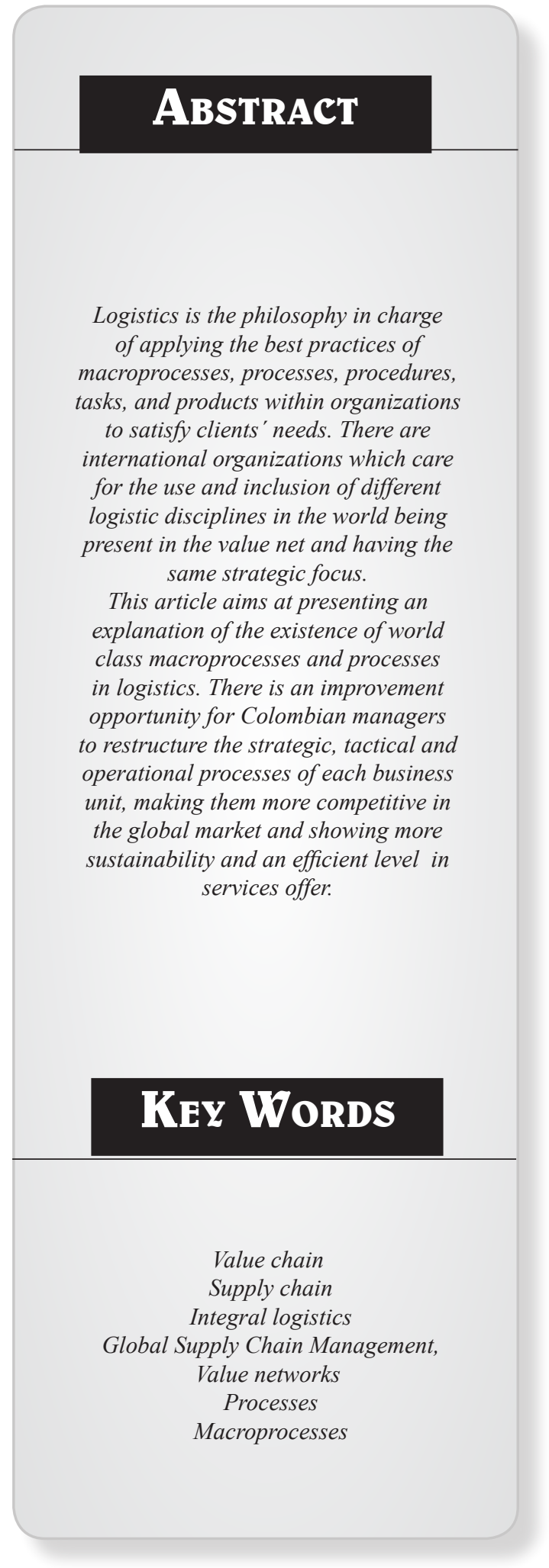


de grandes superficies, minoristas, turismo, empresas de bienes, servicios y otras.

La logística es llamada por varios autores, logística de los negocios, haciendo énfasis en sistemas de respuesta rápida al cliente, administración de los canales de distribución o entrega, logística industrial, distribución física internacional, administración de la cadena de suministros y actualmente red de valor.

La logística tiene varios entes que velan por las buenas prácticas en el quehacer logístico en el mundo, uno de los cuales es el Council Logistics Management Professionals(CSCMP), que estáubicado en los EEUU, que antes se denominaba Council Logistics Management (CLM). En el ámbito Europeo se encuentra la
European Logistics Asociation (ELA) que reúnen los líderes logísticos en cada uno de los continentes, realizando las mejores prácticas que en este trabajo se pretende evidenciar con sus experiencias logísticas.

Estos dos entes tienen más de 10.000 miembros alrededor de todo el mundo con un propósito principal de generar inquietudes para los líderes de la logística integral en las organizaciones, aplicando filosofías de última generación y estas buenas prácticas logísticas se difunden a través de sus miembros por medio de casos empresariales de estudio y crean un gran interés en los diferentes niveles de la empresa extendida que impacta el desempeño logístico.

\section{Gerencia de la cadena de abastecimiento global o (global supply chain management)}

\subsection{Alcance del global supply chain management}

Son incalculables los retos a los cuales las empresas y organizaciones deben enfrentarse hoy en día. No basta sólo con hacer las cosas bien, es necesario ser excelente en el quehacer logístico local, nacional e internacional.
Lacreciente competitividad organizacional y los fenómenos de la globalización y la internacionalización de los mercados, a los cuales nos enfrentamos en la actualidad exigen, de las empresas, respuestas cada vez más eficientes, con enfoques en macroprocesos, procesos y estrategias logísticas, que les permiten sobrevivir y crecer en un mundo en 
continuo cambio, en el cual el cliente es cada vez más exigente, con grandes posibilidades de negociación, y quien, al final, define el éxito o fracaso de todo el engranaje empresarial que se activa con el planeamiento de los recursos basados en las necesidades de los clientes o para reducir el impacto a un problema existente en la organización que tarde o temprano impactará en la sociedad como el suministro de materiales e información, fabricación de un producto, entrega al consumidor final y un óptimo valor agregado del producto en las manos del cliente.

La logística como filosofía integradora de procesos y la cadena de abastecimiento no deben conformarse con entregar el bien o servicio al cliente, sino que deben determinar que tan satisfecho se encuentra el cliente final, y cuando al consumidor se le ha llenado sus expectativas, se afirma que se ha realizado un óptimo trabajo en la organización donde se ha cumplido con la misión que es identificar las necesidades del cliente y buscar cómo suplir los deseos del consumidor final, y que todos los eslabones estén orientados hacia un mismo fin y hacia una misma estrategia.

En la última década se ha determinado que las empresas son conscientes de que, a medida que avanza el tiempo, cada cliente individual cobra más importancia, y que para satisfacerlo no basta con que una de las organizaciones que colaboran en el desarrollo de los productos se hagan de una manera óptima; un producto será de buena calidad, será competitivo, solo si ha pasado por procesos de mejoramiento continuo buscando la excelencia a lo largo de toda la cadena de suministro con buenas prácticas logísticas (Global Supply Chain Management) generando un valor agregado al producto a los procesos donde se fortalece cada vez una red de valor.

Si se analizan los índices de desempeño logístico del país, se observa que se requiere de un alto porcentaje de plan de contingencia o de acción, con el fin de atacar los diferentes sectores y regiones que están vulnerables a las exigencias actuales, ya sea con los tratados de libre comercio u otros, para unos clientes cada vez más exigentes.

Según un estudio de World Bank ${ }^{1}$, "Colombia ocupa el puesto 82 del ranking de una muestra de 150 países, que al compararlo con los países de la región ocupa el penúltimo lugar, ubicándose por debajo del promedio de Suramérica, e incluso de Latinoamérica y el Caribe", este tipo de mensajes demuestran que Colombia tiene una gran oportunidad de mejora con el fin de desarrollar día a día la infraestructura y la competitividad

Fuente: Connecting to Compete. World Bank, 2007 
acercándose cada vez a mayores inversionistas de empresas trasnacionales que ayuden a generar mayor empleo y desarrollo para los diferentes sectores económicos en este caso se debe determinar muy bien el alcance en el momento de diseñar, planear e implementar la red que va apoyar el Global Supply Chain Management.

La teoría de las restricciones. formulada por Eli Goldratt, en el libro La Meta, es una filosofía que se debe contemplar en el momento de planear los recursos de los eslabones de la cadena de suministro global que se basa en la capacidad de los procesos y se enfoca en analizar cuáles son los recursos que poseen la menor velocidad (cuello de botella) para plantear el modelo específico para el óptimo funcionamiento de todos los involucrados del sistema con el propósito de identificar las restricciones.

En la actualidad el Global Supply Chain Management está orientado a sistemas de producción pull, que se encuentra sincronizado con la información de la demanda del consumidor que el sentido de dirección está dirigida desde el cliente hacia el proveedor determinándose de la siguiente manera: primero, gestión de los retornos, segundo entrega (distribución), tercero producción, cuarto abastecimiento y finalmente la planeación.
La pregunta que se realiza frecuentemente el personal que está directamente o indirectamente involucrado en la empresa, sería entonces ¿Qué es la cadena de suministro? ¿Es lo mismo que la logística integral? y ¿Cuál será su diferencia?

Lo primero que se debe tener en cuenta es analizar y conceptualizar lo que es la logística, la logística integral y la cadena de suministro global con buenas prácticas o el concepto del (Global Supply Chain Management), y tener claramente identificados los macroprocesos y procesos de la cadena de abastecimiento de clase mundial como lo enuncian los autores. (Avantage S. Ch. Y Shoshananah C. y J., 2005).

El alcance del Global Supply Chain Management está comprendido como se observa en la gráfica anterior desde el proveedor del proveedor, hasta el cliente del cliente, generando todo un engranaje organizacional que se encarga de satisfacer las necesidades del cliente dentro de un aspecto local, nacional e internacional, con un óptimo retorno de la inversión, diversificando nuevos productos a través de todos los continentes y generando no solamente valor agregado sino enmarcado en un ámbito colaborativo entre vendedores y compradores, con impacto social y variables geopolíticas que son necesarias contemplar entre todos los eslabones involucrados. 
Alcance del Global Supply Chain Management

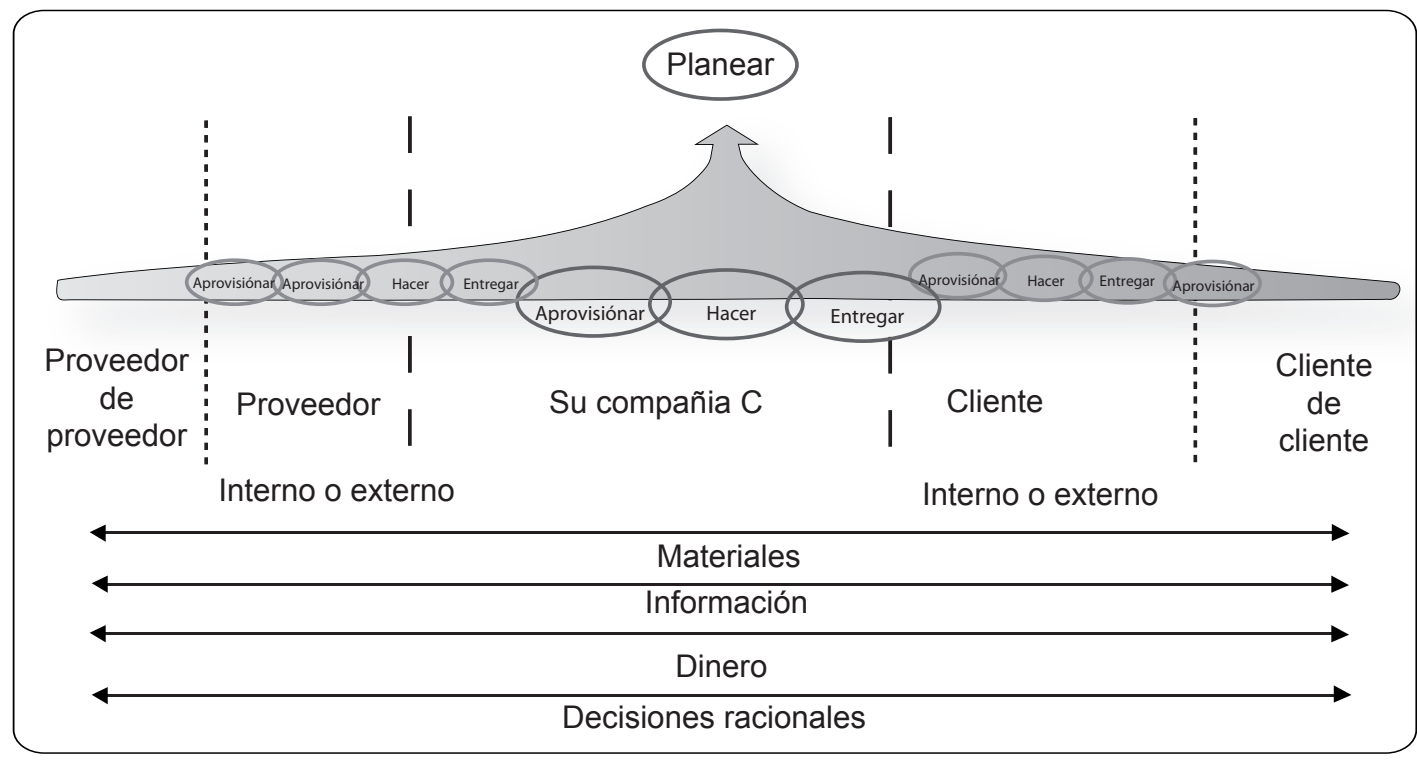

Fuente. Copyright 2001 Supply Chain Council, inc.

\section{Conceptos básicos}

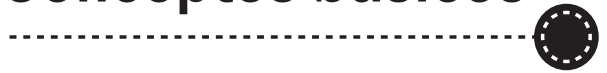

L

exigencia actual por satisfacer las necesidades del cliente está a la orden del día en las compañías y organizaciones que desean ser eficientemente productivas, además que desean permanecer en la competencia global en nuevos mercados y la sincronización de procesos desde el proveedor del proveedor hasta el cliente del cliente buscando una mejora continua del desempeño logístico.
Los entes tales como el Council Supply Chain Management Professional de EE.UU (CSCMP) y la European logistics Association (ELA), han determinado claramente las operaciones a desarrollar por los macroprocesos y procesos del Global Supply Chain Management de clase mundial los cuales se enuncian a continuación y que son cruciales para el éxito de la red de valor. 


\section{Macroprocesos de clase mundial del global supply chain management}

Los autores del libro Supply Chain Management, (Chopra S. y Meindl P., 2007) enuncian que la cadena de abastecimiento se basa en tres macroprocesos que están configurados de la siguiente manera: primero, la Gestión de Proveedores (Suppliers Relationship Management) SRM, define claramente que hay actividades tales como : el aprovisionamiento, negociación entre proveedor -cliente, gestión de compras, diseño de colaboración (proveedor cliente), el segundo macroproceso, es denominado Internal Supply Chain Management (ISCM), por sus siglas en ingles, en este se determina la estrategia integradora y coordinada del negocio, incluyendo el planeamiento estratégico, planeación de la demanda, planeación de proveedores, el énfasis hacia el servicio al cliente, la utilización de estrategias de outsourcing como ejemplo la utilización de maquilas y producciones in house y el tercer macroproceso, denominado gestión de relaciones con el cliente (Customer Relationship Management) C.R.M, donde se realizan actividades tales como: la administración de la orden del cliente, Call Center, indagar las últimas tendencias de los clientes, venta de productos, retroalimentación del nivel de servicio en la cual se deben tomar los correctivos del caso y si hubiese una operación no conforme se realice un plan de contingencia para tomar decisiones al respecto y solucionar el problema presentando en la cadena de suministro global.

Macroprocesos clase mundial del Global Supply Chain Management

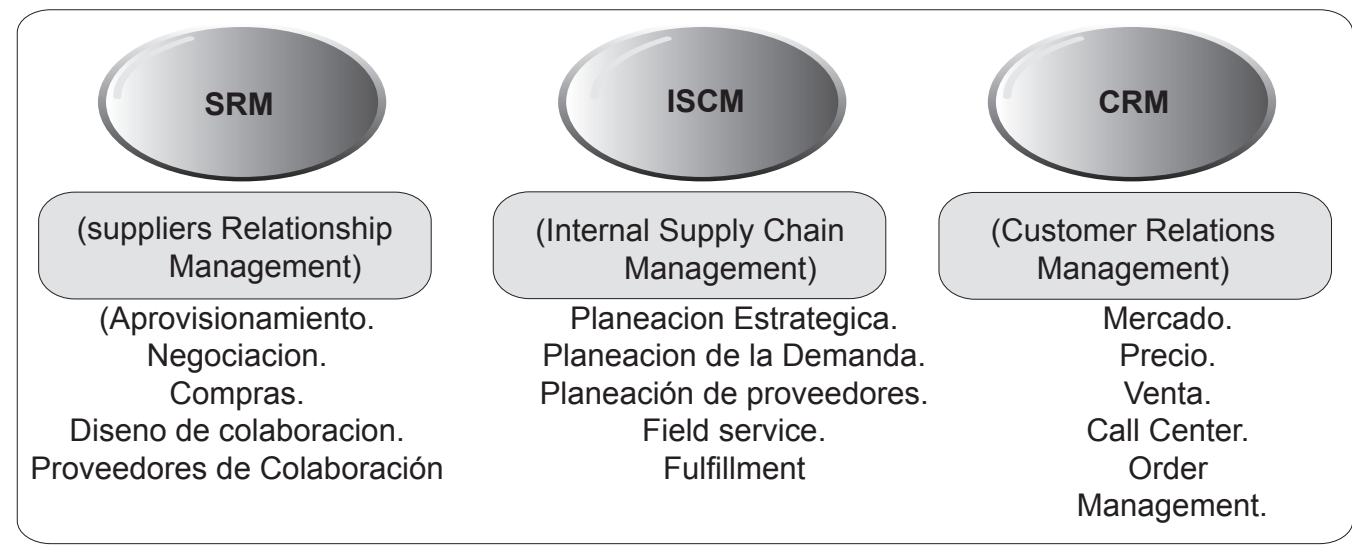

Fuente. Chopra Sunil y Meindl Peter, Supply Chain Management; Strategy, Planning and Operations - 3rd Edition.2007.New Jersey, editorial Prentice Hall. 


\section{Procesos de clase mundial del global supply chain management}

Los autores del libro en francés denominado Avantage Supply Chain (Cohen S. y Roussel J.,2005), determinan cinco procesos claves de la cadena de suministro global que es pertinente analizar dentro de una unidad de negocio y se enuncian a continuación: la planeación, el abastecimiento, la producción, la entrega y la gestión de los retornos. En Colombia existe una gran oportunidad de mejora para que las organizaciones que trabajan en la administración de la logística apliquen los siguientes procesos contemplados de clase mundial por los entes que lideran la logística tales como la European Logistics Asociation ELA, Council Supply Chain Management Profesional CSCMP y que actualmente más del $60 \%$ de las empresas en Colombia carecen de procesos integrados a la hora de aplicar sistemas integrados informáticos tales como un ERP (Enterprise Resource Planning ) como expresa el autor Andres Muñoz (2005), en la cual muchas empresas que implementan el sistema llegan a final termino debido a varias razones, una de ellas es el cambio de cultura organizacional, dos bajo compromiso de la gerencia, entre otras.

Para explicar más detalladamente cada uno de los anteriores procesos se describe a continuación.

\subsection{Planeación}

Se debe basar en todo lo necesario para cumplir con los requerimientos del cliente y se planea la infraestructura necesaria para cada uno de los eslabones que intervienen en la cadena de suministro. Conocer perfectamente las mejores prácticas en el dominio de Supply Chain, involucrar personas de funciones diferentes que hagan bien su labor a fin de trabajar en colaboración con todos los integrantes de la cadena de valor además de localizar las oportunidades de mejoramiento y automatización de procesos. Es indispensable utilizar informaciones en tiempo real, dirigida a los recursos y prioridades comerciales, que apunte a la simplificación de la operación, con el propósito de generar integración de las necesidades en términos de Supply Chain (aprovisionamiento, producción, entrega, gestión retornos), asignar tareas y responsabilidades. 
Actualmente existen estrategias y sistemas informáticos competitivos que ayudan a la planeación de la logística como es el APS Advanced Planning Systems que son modelos matemáticos que se ajustan a todas las necesidades de los eslabones de la cadena de suministro global con el fin de minimizar el riesgo potencial que puedan tener los procesos y centralizar la información pertinente para la óptima toma de decisiones.

\subsection{Abastecimiento o suministros}

Son las gestiones y acciones frente a la integración proveedores - clientes con base en los lineamientos de gestión de compras y el suministro de materiales, información, entre otras.

Este proceso se refiere a la gestión de las actividades y todos los servicios necesarios para ejecutar las actividades de compras, orden de pago, recepción, inspección y autorización de pago a proveedores. Los principios básicos son el costo total de posesión, se deben definir estrategias por categorías $A B C$, la selección y certificación de proveedores, crear indicadores de gestión o métricas o key performance indicators (KPIs) para medir la gestión del proceso de abastecimiento donde muchas organizaciones lo tiene inmerso dentro del proceso de compras.

\subsection{Producción}

Es el eslabón que se encargará de lafabricación o la transformación física de las materias primas hasta producir el producto.

Para obtener procesos eficaces de producción se deben concentrar las prioridades comerciales teniendo en cuenta la flexibilidad, la efectividad del proceso, el costo de la hora de fabricación y velar por el cumplimiento de las normas de calidad, la sincronización de las actividades de operación.

\subsection{Entrega}

Es la gestión de distribución del producto o el bien y el aseguramiento del suministro al consumidor final.

El proceso de entrega comienza en el momento en el cual la empresa recibe una orden del cliente y la entidad cubre todas las actividades permitiendo ejecutar y gestionar la orden. Un proceso de entrega eficaz debe aplicar las siguientes actividades; primero, encontrar el buen equilibrio entre el nivel de servicio y su costo; segundo, reducir los costos y los plazos por medio tratamientos simples y rápidos; tercero, colocar los productos en el sitio donde sea requerido por el cliente seguido de una óptima trazabilidad transversal y administrar los datos propendiendo garantizar las entregas exactas justo a tiempo. 


\subsection{Gestión de los retornos - logística inversa}

Este proceso es muy importante porque debe cumplir con los requerimientos de la logística verde e inversa, y la idea es que todas las materias primas cumplan con los lineamientos de la ISO 9001,14001,18000, 26000,28000 y generar el plan de contingencia para devolver el producto o el bien de nuevo a la cadena de suministros, para realizar los cambios respectivos si se llegará a presentar algún defecto, retroalimentar al sistema nuevamente como administración de lecciones aprendidas (ALA) además de conocer las últimas tendencias del consumidor final con el fin de tener en cuenta estas tendencias y retroalimentarlas al proceso de planeación nuevamente.

Este proceso garantiza que los productos vendidos sean administrados y recogidos según las políticas de la empresa y los acuerdos con los clientes.

Para realizar un proceso de gestión eficiente se debe realizar principalmente una estrategia integradora en sentido contrario al usual cliente -proveedores, comunicándose en tiempo real con las informaciones de retorno, determinando las políticas en función del costo total.
Las buenas prácticas lideradas por los entes internacionales tienen claro que las empresas, compañías y organizaciones deben velar por una estrategia que los integre por medio de procesos transversales cliente -proveedor donde todos los involucrados manejen las mismas estrategias y con el mismo fin.

Cuando hablamos de integración de redes de valor o Global Supply Chain Management no es una tarea fácil, pues estamos refiriéndonos a la unión o integración de múltiples empresas, con las implicaciones que esto conlleva; diferentes culturas, tecnologías, macroprocesos, procesos, entre otras, que deben unirse y trabajar como uno solo y de la mejor manera posible y por un solo objetivo la satisfacción del cliente.

La ventaja competitiva actualmente es entre cadenas de abastecimiento y no solo entre empresas, donde el trabajo en equipo debe prevalecer todo el tiempo y solamente va ver dos tipos de empresas, una la que está en proceso de cambio y segundo, las organizaciones que están en proceso de desaparecer enuncian el autor como (Philips Kotler,) con una mentalidad prospectiva en función de la logística con el fin de innovar procesos y reducir tiempos de entrega (lead time) en función de satisfacer el consumidor final. 


\section{Estrategias}

na estrategia clave indispensable para lograr y alcanzar un buen proceso de Gestión del Global Supply Chain Management es, importante hacer un óptimo proceso de gestión e integración logística en el interior de cada una de las empresas que conforman la red; no se puede propender alcanzar altos niveles de desempeño si cada organización acepta ineficiencias operativas, administrativas y no tiene el concepto de macroprocesos y procesos, subprocesos, actividades y roles logísticos claros.

Por lo tanto, el primer paso en la búsqueda de las buenas prácticas logísticas de la cadena de suministros es tener un alto nivel de integración en la logística interna de las empresas, en las cuales deben sincronizarse todos los procesos logísticos como partes interrelacionadas e interdependientes entre sí, de tal manera que se busquen mejoras siempre enfocadas hacia el canal en general y no sólo para cada elemento en particular.

Para tener una estrategia logística dentro del Global Supply Chain Management se deben tener en cuenta los siguientes parámetros:

\subsection{Logística Integral}

A partir de los años 90 ha incursionado en el quehacer logístico terminología avanzada para enunciar los procesos internos y externos de la logística. En la actualidad se mencionan dos clases de logística, una interna (Inbound) (Grant D. et.al. 1998) que refiere a la administración eficiente de los flujos de los materiales y productos en la compañía tal como procesos en la producción, distribución, transporte y logística externa (Outbound) que está orientado a la logística de almacenaje y transporte orientado a la parte final de la cadena es decir esto es el cliente, basado en información de la demanda.

Desde el punto de vista interno, la logística integral se refiere al concepto tradicional de costes totales, donde se analiza " la gestión sincronižàda de cada uno de los integrantes de la cadena de suministros tal como son los procesos del Global Supply Chain Management de clase mundial tal como son: la planeación ,abastecimiento, producción , entrega y Gestión de los Recursos (Logística Verde y logística Inversa), donde debe existir unas actividades muy claras en cada uno de los integrantes y un balance entre la logística y el marketing para no 
incurrir en agotados con bajo nivel de servicio al cliente o altos inventarios y obsolescencia de productos dentro de los procesos y almacenes.

Todos los productos tienen cadenas de abastecimiento pero de ahí a tener buenas prácticas logísticas puede ser dudoso de encontrar, la idea radica en tener unos procesos integrados en la cadena de suministro global que en nuestra cultura ha sido difícil de asimilar integrando proveedores a la red requiriendo buenas prácticas en la gestión ascendente de la unidad de negocio y la clasificación prioritaria de clientes $A B C$ que también no es muy fácil de evidenciar.

Unos de los problemas más representativos para la organización colombiana es que no se piensa de manera global, holística e integral dentro de los procesos de la compañía y hay grandes debilidades frente a la cultura de administrar el riesgo en los procesos organizacionales, debido a que siempre que se piensa en riesgo se viene a la mente riesgos en seguridad industrial, considerada como una pequeña parte de la gestión del riesgo dentro de la cadena de valor. Una cosa ha sido la logística antes de los hechos ocurridos el 11 de septiembre del año 2001 en EEUU, debido a los diferentes errores cometidos en los procesos de las organizaciones y otro es la logística después de este evento, es por esto que la organización debe centrar su atención en cómo medir la vulnerabilidad de cada uno de sus procesos organizacionales y de todos los procesos que impacten la cadena de abastecimiento, además se está observando cada día más la preocupación del impacto de los productos y servicios al medio ambiente lo que ha generado que entes $y$ empresas trabajen por la reducción de las emisiones de dióxido de carbono a escala global y en cada uno de sus procesos internos y externos de la cadena de valor propendiendo una economía en la producción de energía que es una de las mayor fuentes de índices de carbono a la atmósfera y al fenómeno invernadero. La Gestión del Riesgo como lo determina los entes tales como COSO, (Committee of Sponsoring Organisation) de los EE.UU y COBIT (Control Objectives for Information and related Technology) en la cual se enfocan en reducir, mitigar y transferir el riesgo, a todos los procesos de la compañía incluyendo los derechos humanos y al derecho internacional humanitario.

La logística enmarcada dentro de una filosofía de reducción de riesgo, está relacionada con todas las áreas de la empresa y el propósito del trabajo con equipos polivalentes es que aporten a la efectividad de los flujos ya sea de materiales, información, recurso humano y toma de decisiones. 
Distribución fluida: una manera de determinar el desempeño logístico de la cadena de suministro global, es validar como se encuentra el recurso humano, flujo de información, materiales, decisiones administrativas por todos los integrantes de la cadena de suministro, con el propósito de utilizar sistemas de información en tiempo real, con base a la información de la demanda del consumidor y una respuesta rápida al requerimiento del mismo.

Los parámetros que se deben analizar en la distribución fluida son:

$>$ Costos: el costo de fabricar y mover los materiales desde el proveedor o proveedor del proveedor al consumidor final.

Servicio: es un indicador muy importante dentro de la logística porque evalúa el desempeño en la entrega de producto o el bien al cliente, indica además el porcentaje de agotados de los productos y el lead time (el tiempo desde que se genera la orden del requerimiento del cliente hasta el momento de la entrega al consumidor final) además de determinar que tan satisfecho se encuentra el cliente con el producto o bien adquirido dentro del Global Supply Chain Management.

D La visibilidad de los inventarios: este tipo de criterio es clave para conocer donde se encuentra los productos y procesos en la cadena. Las operaciones fluidas necesitan una visión de los indicadores de utilización de producto por medio de (Key Performance Indicators o metrics) a través del canal de suministro que prevengan, detecten, controlen y corrijan los riesgos dentro procesos de la organización.

$>$ Las organizaciones que tienen buenas prácticas miden por medio de indicadores el grado de utilización de sus productos, por ejemplo en las bodegas de materia prima, en productos en proceso y terminado, en las ventas se deben tomar decisiones para sincronizar sus procesos operativos, tácticos y administrativos donde deben ser flexibles y personalizar al requerimiento del cliente, donde es pertinente medir la eficiencia y eficacia que impacta en los consumidores finales.

Da velocidad: es el tiempo que lleva movilizar los productos o la información a través de los diferentes procesos y eslabones del Global Supply Chain Management y está directamente ligado, con los niveles de inventario y con la flexibilidad para responder a los cambios repentinos del cliente o de los mismos procesos.

Destionar el flujo: la administración de los inventarios debe estar dentro del 
contexto de gestionar el flujo de la información, recursos, para optimizar el lead time de los productos y los procesos necesarios para llenar las expectativas de los clientes interno y los usuarios finales.

D La cadena de suministro global, debe estar contemplada dentro de una filosofía de administración por procesos donde se utiliza el modelo de caja negra, donde se introduce información o materia prima luego se transforma físicamente y finaliza con la entrega de un resultado ya sea como cliente interno o externo de la organización y donde se debe observar una trazabilidad de los procesos y las actividades del Global Supply Chain Management.

$>$ Distribución flexible: las operaciones de distribución están configuradas de tal manera que sean rápidas y flexibles en la aplicación de diferentes modelos de procesos de Crossdocking y envíos directos entre plantas, juegan un papel muy importante en las operaciones fluidas, para lo cual se debe tener una infraestructura de entrega (Distribución) cada vez más rápidos y efectivos combinando diferentes estrategias de transporte Intermodal y multimodal.

> Fabricación con base a Just In Time (JIT): la gestión de operaciones fluidas necesita que los productores realicen cambios rápidos y repentinos con el fin de obtener ediciones de producción más cortas. Otro criterio que tiene esta filosofía es que la manufactura aplica conceptos de control de calidad asegurando el abastecimiento de materiales e insumos a los procesos de producción evitando el riesgo de agotado y con el fin de minimizar el tiempo obsolescente por errores en la planificación de los recursos.

D Los fabricantes deben ser lo suficientemente rápidos y flexibles para responder a los cambios de la demanda y de los requerimientos de los clientes, dentro de un marco de filosofías de planificación de capacidad que involucren el fenómeno de fin de mes, tal como sucede con los picos de la producción que genera errores e insatisfacción al cliente interno y externo, debido a las entregas de los pedidos de los productos al final del periodo. Dos grandes preocupaciones son permanentes en los responsables industriales, lo primero es aumentar la reactividad de los sistemas logísticos (entregar sin plazo una demanda diversificada), de otra parte, disminuir los costos totales de producción eliminando gastos y la operaciones inútiles que no agreguen valor a los procesos como al producto.

Es vital para un óptimo desempeño de la logística un sistema de información que 
soporte a las diferentes áreas, procesos y macroprocesos de la cadena de aprovisionamiento global pero hay que resaltar que el Sistema de Información si procesa datos basura genera como resultado datos de salida que no agregan valor, es por esto que se debe alimentar y parametrizar el sistema con datos reales y a tiempo para alimentar los Indicadores de gestión.

\section{EI GSCM}

P ara enfrentar la competencia a nivel mundial hay cuatro desafíos claves para ser un Global Supply Chain Management de clase mundial son: primero, el énfasis hacia el consumidor final, segundo, utilizar estrategias de outsourcing para procesos de transporte, almacenaje, logística inversa y verde entre otros, tercero, busca incursionar al desarrollo de nuevos productos y nuevos mercados en otros continentes y cuarto, utilizar sistemas de información de última generación para controlar y gestionar los datos reales de la organización².

Busca que las organizaciones hayan hecho todo por mejorar y optimizar sus operaciones logísticas a nivel interno y externo donde se debe evaluar el desempeño de cada uno de los procesos y macroprocesos de la misma, donde el objetivo es mejorar la competitividad, la rentabilidad, la prospectiva tecnológica, reducir el riesgo de los procesos y su principal fin la satisfacción del cliente.

La estrategia de generar una verdadera red de valor consiste en que todos los miembros estén orientados hacia un mismo propósito con el fin de integrar (Proveedores de Proveedores fabricantes-productores-distribuidoresmayoristas - minoristas, clientes y clientes de clientes).

D Orientado al cliente: Se debe recordar que el Global Supply Chain Management se activa en el momento que se genera el requerimiento del cliente y con base en esta necesidad, se planea los recursos para los procesos de abastecimiento, producción, entrega y gestión de los recursos (logística verde e inversa).

Recordemos que la cadena de abastecimiento debe comenzar a fluir desde el mismo momento en que se genera la orden del cliente, es por está razón que el último proceso de la logística denominado gestión de los retornos vela por las actividades de servicio al cliente caracterizados por la satisfacción

Fuente:, Actores Perret Francis-Luc, Jaffeux Corynne, Wieser Phillipe, and Fender Michel, Essentiels Of Logistics and Management,Dominio editor Management of Technology, Collection EPFL-PRESS, ISBN 978-2-940222-16-2,ano 2007, Switzerland. 
de las necesidades del consumidor y si no se tiene claro este concepto la competencia va aprovechar la debilidad y podría convertirse en un alto riesgo, denominado de desventaja competitiva donde las empresas que sean efectivas y proactivas se llevaran los clientes.

\Gestión de los activos logísticos: $\mathrm{Si}$ se pretende tener un óptimo nivel de servicio al cliente, ¿En qué deben basarse las decisiones?, conociendo las repercusiones que éstas tendrán, en las otras empresas de la red de valor.

Cuando se planean proyectos de logística se debe tener en cuenta el diseño de los canales de entrega, localización de instalaciones de distribución , operaciones de transporte, inventarios en proceso, entre otros; toda la infraestructura debe configurarse dentro de los parámetros de las necesidades de cada uno de los integrantes y eslabones de la cadena, además de compartir la información para hablar el mismo idioma obteniendo mayores beneficios globales y, en segundo, lugar, establecer los criterios principales de funcionamiento y la participación de cada empresa en el desarrollo del proyecto, determinando claramente quién hace qué.

La empresa debe decidir con base en sus objetivos, políticas, mercados, clientes, tipos de producto, capacidades y costos, entre otras variables, qué indicadores de control debe tener sobre los canales de entrega o distribución.
No todas las empresas tienen el mismo modelo de aprovisionamiento para los diferentes tipos de bienes, materia prima, información, para la transformación de diferentes tipos de productos; de la misma forma y manera, la demanda de los bienes a través de diversos canales también exige diferenciación en cuanto a la configuración de los pedidos -cantidad y variedad, los tipos de empaques a utilizar, el tipo de transporte y los tiempos de llegada al mercado (Lead -time).

Para efectos de estrategias de outsourcing para el proceso de entrega (distribución), existen varios niveles de operación de los operadores logísticos, la decisión de utilización de two party logistics (2PL) para operaciones de almacenaje y distribución, three party logistics (3PL), estos servicios pueden ser administrativos como operativos o ambos, además de incluir más que un servicio de transporte o almacenamiento y fourth party logistics (4PL), se caracteriza por ejecutar la planeación y coordinación desde los proveedores hasta los clientes, importando y exportando productos en otros continentes, enfrentando la globalización e internacionalización de mercados y la ultimo tendencia con operadores logísticos denominados lead logistics provider (LLP), que se caracteriza por la integración con otras redes de valor, prestando servicios de consultoría para la planificación e implementación del uso de varias cadenas de abastecimiento con las ultimas herramientas de E-business. 
La idea de crear sociedad y modelos de colaboración con los diferentes operadores logísticos, maquilas, producciones in house hacen que haya actividades especializadas en cada uno de los eslabones de la cadena de abastecimiento, reduciendo el riesgo y generando alianzas estratégicas (joint venture), compartiendo información, infraestructura y trabajando de una manera integral con la empresa que lidera el Global Supply Chain Management .Coordinación de gestión de las relaciones con el consumidor: En esta variable donde el consumidor debe y quiere observar la trazabilidad del proceso de fabricación o conocer cuándo se le irá a entregar su producto, siendo de suma importancia que haya una óptima visibilidad del personal, recurso y proceso dentro del Global Supply Chain Management. Es importante establecer indicadores de gestión o sistemas que alerten cómo está el nivel de servicio en el consumidor tal como es una estrategia o sistema denominado ECR (Efficient Customer Response), y actúa también como indicador que mide la eficiencia, eficacia y efectividad en la red de valor y que tan integrado se encuentra la gestión cliente-proveedor.

> Integración del área de ventas y la planificación de operaciones:

El pronóstico de la demanda es la información base más importante donde se determinará el comportamiento de las ventas y del consumidor final.
Determinado que los engranajes de cadena de abastecimiento global son sus procesos y macroprocesos, donde la orden del cliente se encarga de halar el requerimiento o necesidad desde la materia prima hasta la entrega final del cliente satisfaciendo la necesidad del consumidor.

Existe una herramienta tecnológica denominado a Point of Sale (POS) donde el ejercicio consiste en realizar un seguimiento continuo y real del inventario determinando que el fabricante pueda observar las tendencias de la demanda, anticipando y sincronizando los eslabones tal como la producción, mejorando el desempeño logístico, optimizando el inventario y dando mayor flujo de productos, recursos y un rápido retorno de la inversión.

D Estrategias de modelos de colaboración: existe un modelo denominado de colaboración donde sus siglas en ingles son CPFR (Colaboration, Planning, Forecasting,Replenishment), este modelo consiste en crear un lazo logístico entre los integrantes del Global Supply Chain Management, generalmente entre proveedor -cliente donde se asegura el suministro oportuno de materias primas o información a un precio óptimo, en el lugar indicado, con los estándares de calidad pertinente, en la cantidad indicada evitando inventarios obsolescentes, 
incremento de los costos ocultos, baja rentabilidad, alto nivel de riesgos en sus procesos, incremento en costos de mantener, costos ordenar y entre otros.( Chopra S. y Meindl P.; 2007).

$>$ Desarrollar indicadores de gestión para los consumidores: las métricas, indicadores de desempeño o en las siglas en ingles de Key Performance Indicators (KPIs) son medidas bastante útiles para valorar la eficiencia de la empresa y la cadena de suministro, sin perder el concepto que lo que no se conoce no se mide, lo que no se mide no se controla y lo que no se controla no se mejora.

\section{Ventajas del Global supply chain management}

Las ventajas de una gestión de la cadena de abastecimiento global con buenas prácticas son innumerables, pero resaltamos las más representativas:

D Plazo adaptado a los pactado proveedor-cliente.

$>$ Flujo ágil de productos y servicios.

D Una respuesta rápida al cliente y a la toma de decisiones.

D Reducción de Inventario de materia prima, producto en proceso y producto terminado.

$>$ Reducción quejas y devoluciones.
$>$ Mejor servicio al cliente y nivel de servicio.

$>$ Mayor disponibilidad de los bienes.

$>$ Reducción del riesgo organizacional con base a programas de administración del riesgo en procesos administrativos y operativos.

> Óptimo modelo de compras adaptado a cada tipo de organización.

D Visibilidad y trazabilidad de productos, roles, actividades, procesos y macroprocesos en todo el Global Supply Chain Management. 
D Óptima sinergia entre los diferentes procesos y áreas de la organización, enfocada en administración por proceso dejando atrás la gestión funcional.

D Reducción de tiempo desde la orden del cliente hasta el consumidor final.

D Flujo oportuno de productos y servicios.

D Aumento del valor de los productos, procesos, en las redes de valor.

$>$ Incremento de la rentabilidad y reducción de costos.

En la gestión logística es oportuno realizar y analizar las siguientes preguntas acerca de cómo está el Global Supply Chain Management de la organización:

- ¿Qué entiende por la logística integral?

- ¿Conoce la estrategia del Global Supply Chain Management y los beneficios que trae frente a los tratados de libre comercio?
- ¿Se han realizado modelos de colaboración dentro de la organización para acercar a proveedor-cliente?

- ¿Conoce claramente los últimos requerimientos de sus clientes y los tiene clasificados por costos $A B C$ ? ¿Conoce la vulnerabilidad y la protección existente de los procesos organizacionales?

- ¿Ha pensado en diseñar nuevos productos o renovar los actuales, si va abrir nuevos mercados?

- ¿Conoce en qué porcentaje está el nivel de servicio del Global Supply Chain Management en la empresa?

- ¿Tiene planes operacionales, tácticos y estratégicos en la cadena de suministros?

- ¿Tienela cadena de abastecimiento un valor agregado frente a los productos, procesos? y ¿Tiene un plan de contingencia en caso que se cometan errores? ¿Selecciona y evalúa proveedores? 


\section{Megatendencias logísticas}

El mundo competitivo cada vez está siendo más exigente a los requerimientos del cliente, actualmente los consumidores requieren que sus cadenas de abastecimientos tengan:

$>$ En el proceso de servicio al cliente debe contener mínimo las actividades de la administración de la orden del cliente tales como son: el antes de generar la orden del cliente denominado el proceso de (pretransacción), el durante de la (transacción) y el después considerado la (postransacción), lo cual se utiliza por medio de herramientas de intercambio electrónico de datos denominado (EDI).

$>$ Catálogo de los productos por medio electrónico dentro de la filosofía E-Business.

D Utilización de herramientas tecnológicas tales, como el código de barras combinándolo con el código electrónico del producto (EPC), aplicando técnicas de trazabilidad RFID para los productos, procesos, materiales y la logística inversa en toda la cadena de abastecimiento con el fin de brindar mayor trazabilidad y visibilidad a los procesos, productos y cumplir con la exigencia de los clientes.

$>$ Un valor importante dentro de la gestión por procesos es tener certificaciones y la aplicabilidad de las normas dentro de los procesos enfocados en los sistemas de gestión de calidad y buenas prácticas internacionales en recurso humano y gestión del riesgo, tales como son: la ISO 9001, que se refiere a la certificación de procesos de calidad, ISO 14001, se basa en la relación óptima del producto con el medio ambiente, OSHAS 18001, referente a la gestión del recurso humano, administración del riesgo acorde a las normatividades (As-Nz 4360) y Administración del riesgo para los sistemas de información basados en las (IEE17799/ISO27001) y ISO 26000 , referente a la gestión social de la cadena de abastecimiento.

> La operación de la cadena de abastecimiento, funciona, 7 dias a la semana, por 24 horas al día y los 365 dias al año.

$>$ Gestión de compras y aprovisionamiento entre proveedor- cliente, 
utiliza herramientas virtuales tal como es el E-Business, E-Procurement, E-Sourcing, Business to Business, $B 2 B$, Business to Commerce, B2C y Business to Government, B2G. Estas estrategias y sistemas apoyan la gestión arriba de la cadena de abastecimiento refiriendo a la (fuente), con el propósito de generar estándares y protocolos, que velan que los recursos de compras, ya sean materiales o proveedores, tengan la suficiente calidad para mantener la empresa extendida, funcionando con un adecuado valor a los procesos y al cliente.

D Para un adecuado desempeño de los recursos empresas con buenas prácticas están implementado un sistema de planeación avanzado denominado APS (Advanced Planning Systems) para configurar la red de la cadena de valor para todos los recursos necesarios disponibles con base a sus restricciones, capacidades de los procesos y el producto final será lo que debe controlar el ERP (Enterprise Resource Planning) a través de los procesos y áreas de la organización.

Los sistemas de información en lo-gística evolucionarán en busca de ES (Expert System), donde alertan a los involucrados de los proce-sos y ayudan a tomar decisiones gerenciales, tácticas y operacionales, reduciendo el riesgo operacional en la cual dan soluciones a problemas en los procesos, actuando de manera autónoma inteligente, como si existiera una persona a cargo donde sencillamente el propio sistema el que toma decisiones, basado en múltiples opciones de solución.

$>$ Para medir el desempeño logístico de la cadena de valor, se deben utilizar herramientas gerenciales de control, basado en los objetivos estratégicos de la organización tal como son primero, el Balanced Score Card (BSC), una segunda, herramienta basada en actividades basada en costos, tal como el A,B,C (Activity Based Costing), priorizando roles y actividades en la empresa, clasificando actividades por niveles determinado cuál debe realizarse dependiendo del parámetro $A, B, C$ y por último el modelo de referencia operacional cadena de suministros denominado (ROCS) o el mismo SCOR (Supply Chain Operations Reference) para medir el desempeño del Global Supply Chain Management y crear un lenguaje común a través de la red de valor con base en sus cuatro dominios de desempeño principal: Plan, source, make y delibery.

D Utilizar tecnología para mejorar la integración proveedor-Cliente con 
plataformas comunes en el eslabón producción como el CAD (Computers Aided Design), CAM (Computers Aided Manufacture) y el CIM (Computers Integrated Manufacture), donde todas las anteriores filosofías como sistemas ayudan a mecanizar los procesos manuales de las operaciones, tecnificando la red de valor convirtiéndola en una cadena de suministro global eficiente.

$>$ Las empresas de aviación y de manufactura automotriz en la actualidad, están aplicando estrategias de mejoramientoeneláreadeoperaciones utilizando filosofías tal como es el MRO (Maintenance Repair Operations), para optimizar actividades en el eslabón aprovisionamiento, producción -distribución, donde se desea mejorar el nivel de servicio como cliente interno de la cadena de abastecimiento, ayudando a sistematizar todo el proceso de mantenimiento, almacenaje de repuestos y mejoramiento en la operaciones de este tipo de rol logístico.

DEn las operaciones de los puertos, los centros de distribución, los procesos de la distribución física internacional, DFI, se está implementando la estrategia de vehículos guiados por computador AGV, (Automatic Guide Vehicule) para agilizar las operaciones y minimizar la mano de obra para reducir los riesgos que puedan realizar los operarios y incrementar la efectividad de los procesos.

D Para terminar este artículo deseo manifestar que la logística debe estar ligada a unos sistemas de información que integre las operaciones internas como externas de la cadena de suministro global, que se caracterice por una plena satisfacción del cliente, utilizando estrategias de Outsourcing que apoyen los procesos de almacenaje, producción y distribución, generando redes de valor, un óptimo retorno de la inversión y contemplar equipos de trabajo entre países para reducir, cada vez las fronteras comerciales y con el fin de generar, cadenas de logísticas más responsables y generando más valor al ser humano como parte fundamental del requerimiento del cliente.

$>$ Para ser competitivos en las cadenas de abastecimiento, se debe velar para los procesos internos como externos de la organización estén orientados hacia la satisfacción del cliente, segundo, se debe aplicar estrategias de outsourcing para delegar procesos un operador logístico realice de manera más especializada y a un costo menor, tercero, es importante buscar la estrategia de no solo satisfacer necesidades locales-nacionales sino buscando los modelos de colaboración con las empresas chinas e hindúes, cuarto la aplicación de 
sistemas de información en cada uno de los eslabones de la cadena de suministro global.

\section{Conclusiones}

L as organizaciones que no estén dentro de la estrategia de logística integral y del Global Supply Chain Management, estarán expuestas a desaparecer debido a que serán compañías no competitivas y que tendrán altos índices de generación de riesgos dentro de sus procesos. Con los tratados de libre comercio no podrán competir frente a los requerimientos cada vez más exigentes de los clientes y de las tendencias de los países donde se desea intercambiar productos, servicios y transferencia de tecnología donde se busquen redes de valor que satisfacen las necesidades para los involucrados en la cadena de suministros globales y para la razón de ser de los eslabones logísticos el cliente con un bajo porcentaje de impacto al medio ambiente y con un alto compromiso social sostenible.

La cadena de valor que disponga de los procesos y macroprocesos claramente definidos, enmarcados en una gestión transversal de procesos se considerarán eficientes y eficaces dentro de la red de negocios que gestionen productos y procesos con sistemas de información en tiempo real, generando una trazabilidad hacia adelante $y$ hacia atrás de todas las actividades logísticas utilizando estrategias de Outsourcing como procesos claves y de apoyo para la entrega Just in time de productos al cliente, incrementando el nivel de servicio en toda la Unidad de Negocio. Finalizando es importante determinar que la GSCM y la logística integral, velan que las unidades de negocio, tengan un retorno rápido de la inversión, trabajando por el crecimiento de los sectores y regiones vulnerables, a las nuevas tendencias de los clientes y la sostenibilidad de los diferentes involucrados de la cadena.

Norma IEEE17799, ISO27000, the Committee of Sponsoring Organizations (COSO) y COBIT (Control Objectives for Information and related Technology). 


\section{Bibliografía}

Baglin G., Bruel O (2007): Management Industriel et Logistique (Concevoir et piloter la Supply Chain), Economica,Paris.

Ballou R. (2007): Administración de la cadena de suministro, quinta edición, Prentice Hall, Mexico, 2006.

Bolstorff P. y Rosenbaum R (2003): Supply Chain Excellent SCOR Model, Edition Amacon, New York.

Cohen S. y Rousse J. L, (2005): Avantage Supply Chain, Editions d'organisation, Paris.

Chopra S. et.al (2007): Management Strategy third edition, editorial Prentice Hall, New Jersey.

Documento Concejo Nacional de Política Económica y Social República de Colombia, CONPES 3547.

Gs1 Switzerland, Logistikmark 2009, Editorial Alemana, Suiza.

Kotler Philips, ACOFI Marzo 2007

Lambert D. et.al (1998): Fundamentals Of Logistics Management, editorial Mc-Graw Hill, EE.UU.

Profesores y consultores de MBA Europeos, MBA, L'essencial du Administration par les meillures professeurs, D'Organisation (Éditions Oct 2008,), PARIS, 2008.

Revista Zonalogistica, Bogota y Medellin. 